\title{
The expression of endothelial and inducible nitric oxide synthase and apoptosis in intestinal ischemia and reperfusion injury under the action of ischemic preconditioning and pentoxifylline ${ }^{1}$
}

Teresinha Regina Ribeiro de Oliveira', Geraldo Ferreira de Oliveira", Ricardo Santos Simões"I, Suellen Maurim Feitosalv, Eduardo Hiroshi Tikazawav, Hugo Pequeno MonteirovI, Djalma José Fagundes ${ }^{\mathrm{VII}}$, Murched Omar Taha vIII

'PhD, Associate Professor, School of Health Sciences, Universidade Federal da Grande Dourados (UFGD), Brazil. Acquisition, analysis and interpretation of data; technical procedures; statistical analysis; manuscript preparation and writing. "PhD, Associate Professor, School of Health Sciences, UFGD, Dourados-MS, Brazil. Acquisition of data, technical procedures, manuscript writing.

I'PhD, Department of Morfology and Genetic, Universidade de São Paulo (UNIFESP), Brazil. Immunohistochemical examinations, analysis of data.

IVPhD, Department Gynecology, UNIFESP, Sao Paulo-SP, Brazil. Acquisition, analysis and interpretation of data; technical procedures.

${ }^{\vee}$ MD, Department Public Health, School of Medicine, Universidade Estadual de Campinas (UNICAMP), Brazil. Acquisition of data, technical procedures, manuscript preparation.

VIPhD, Full Professor, Department of Biochemistry, UNIFESP, Sao Paulo-SP, Brazil. Analysis and interpretation of data, manuscript preparation, critical revision.

VIIPhD, Full Professor, Division of Surgical Techniques and Experimental Surgery, Department of Surgery, UNIFESP, Sao Paulo-SP, Brazil. Analysis and interpretation of data, manuscript preparation, critical revision.

VIIIPhD, Associate Professor, Division of Surgical Techniques and Experimental Surgery, Department of Surgery, UNIFESP, Sao Paulo-SP, Brazil. Conception and design of the study, critical revision, final approval.

\section{Abstract}

Purpose: To investigate the expression of nitric oxide synthase (NOS) and apoptosis associated with ischemic preconditioning (IPC) and pentoxifylline (PTX) in intestinal ischemia (I) and reperfusion ( $R$ ) injury.

Methods: Thirty male rats were assigned to 5 groups: (CG), no clamping of the superior mesenteric artery (90 minutes); (IR-SS) saline + ischemia (30 minutes) + reperfusion (60 minutes); (IR-PTX) PTX + ischemia (30 minutes) + reperfusion (60 minutes); (IPC-IR-SS) 5 minutes of ischemia +5 minutes of reperfusion (IPC) + saline + I(30 minutes)+R(60 minutes); and (IPC-IR-PTX) IPC + PTX + I(30 minutes)+ R(60 minutes).

Results: The application of IPC and PTX showed a significantly lower immunohistochemistry reaction for active caspase-3 $(P<0.05)$ compared to IR+SS. The number of cells immunoreactive to $B C L-2$ was higher in the IR-PTX group ( $>>0.05)$. The NOS-2 expression ( $q R T P C R)$ in the IRPTX group $(P<0.05)$ was higher than the values for the IPC+IR-SS and IPC-IR-PTX groups. The NOS-3 expression was significantly upper in the IPC-IR-PTX group than in the CG $(P<0.05)$, the IR-SS $(P<0.05)$ and the IR-PTX $(P<0.05)$ groups.

Conclusions: The BCL-2 and active caspase-3 showed beneficial effects on PTX and IPC. The expression of NOS-2 and NOS-3 in the IPC and IPC-PTX groups showed no synergistic effect. Key words: Ischemic Preconditioning. Pentoxifylline. Ischemia. Reperfusion Injury. Nitric Oxide Synthase. Apoptosis. Rats. 


\section{Introduction}

Intestinal ischemia-reperfusion injury (IR) is a frequent phenomenon with high morbidity and mortality. It is associated with a number of clinical conditions, including abdominal aortic aneurysm, surgery, cardiopulmonary bypass, strangulated hernias, intestinal obstruction, acute mesenteric ischemia, necrotizing colitis, collapse of systemic circulation (hypovolemic and septic shock), and intestinal transplantation ${ }^{1-3}$.

Interruption of blood supply to the intestine could result in tissue damage. However, the subsequent blood flow restoration may lead to additional injury, referred to as reperfusion injury ${ }^{1-5}$.

Although intestinal IR induced inflammation has been studied extensively in animal models and in humans, the induced inflammatory responses have yet to be characterized ${ }^{1,4-9}$. There is abundant information on the compromising effects of IR on many processes in the intestine, and various protective treatment modalities have successfully been applied to mitigate reperfusion injury in animal models of reperfusion injury of the intestine, such as pharmacologic treatment, chemical agents, and ischemic preconditioning ${ }^{3,4,6-12}$.

Among the available strategies is ischemic preconditioning; since its introduction more than thirty years ago in cardiac surgery $^{9,10}$, ischemic preconditioning has demonstrated favorable effects in numerous other clinical situations or with experimental animals in different organs and tissues or in shock by retarding cell death ${ }^{3,4,6,7,9-16}$. Ischemic preconditioning can be mechanical or pharmacological $\left.\right|^{3,4,6,8,9,11}$. Direct mechanical preconditioning in which the target organ is exposed to brief ischemia prior to prolonged ischemia has the benefit of reducing ischemiareperfusion injury $\mathbf{3}^{3,6,7,9-16}$.
Several reports have described the effects of IPC on the reduction of apoptosis of epithelial cells $\mathbf{s}^{3,6,14,15}$, structural protection ${ }^{3,7,9,12,14,15}$, preservation of intestinal contractility ${ }^{12}$, decreased expression of the transcription factor nuclear-kB (NF-kB), cell adhesion molecules and pro-inflammatory cytokines ${ }^{3,7,15,16}$, diminution of the inflammatory infiltrate ${ }^{3,7,13-16}$, and increased synthesis of nitric oxide $(\mathrm{NO})^{11,14}$. NO synthesis derived from IPC has been associated with tissue protection ${ }^{6,5,9,11,14}$. However, the mechanisms by which IPC protects the intestine following IR are still not fully understood.

In addition to IPC, the use of drugs such as pentoxifylline (PTX), a methylxanthine derivative of theobromine, has been proposed to decrease the deleterious effects of $\mathrm{IR}^{3,8,17-23}$. Protective effects of PTX include the anti-inflammatory actions associated with inhibition of NF-kB activity ${ }^{19-22}$, downregulation of interleukin (IL)-6, tumor necrosis factor (TNF)-alpha synthesis, and decreased apoptosis ${ }^{8,19-23}$ and inhibition of NO production by inflammatory signaling ${ }^{17-23}$. The activity of unspecific phosphodiesterase, an antioxidant, increases intracellular cyclic adenosine monophosphate (cAMP) concentrations, and it has been previously used to treat peripheral vascular disease and intermittent claudication ${ }^{17,18,21}$. However, the pharmacological properties of PTX are not fully understood.

Clinical biomarkers are used to diagnose the conditions of the development or inhibition of apoptosis and the deleterious effects of ischemia and reperfusion. BCL-2 (B-cell lymphoma 2), is a protein encoded in humans by the $\mathrm{Bcl}-2$ gene and is specifically considered an important anti-apoptotic protein. Localized in the outer membrane of mitochondria, it plays a crucial role in promoting cellular survival and inhibiting the actions of pro-apoptotic proteins ${ }^{13,15,23}$. 
On the other hand, caspase is a cysteine protease, and cleaved caspase- 3 , the active form of caspase-3, is well known as a marker for cells undergoing apoptosis; it is now widely accepted that cleaved caspase-3 staining is useful in scoring the apoptotic index ${ }^{13,15,23,24}$. Studies in direct IPC have demonstrated the protective effect of $\mathrm{NO}$ on microcirculation $^{2,9,10,14,15,17}$. NO is a free radical produced from L-arginine by the enzyme NO synthase, which has three forms: endogenous NOS (NOS-3), neuronal NOS (NOS-1), and inducible NOS (NOS-2) $)^{3,5}$. Of these, NOS-3 and NOS-1 are constitutively expressed, while NOS-2 is produced in response to cytokines and NF-kB. NOS-3 has a protective effect on the microcirculation and always produces NO in small amounts, which predominate in the circulation, producing a protective effect on the microcirculation that is of vital significance

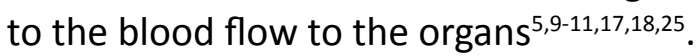

Histological evaluation in our previous publication showed a favorable effect in the IPC-IR-SS and IPC-IR-PTX groups, which is in accordance with the hypothesis of a beneficial effect of IPC alone or PTX; however, the simultaneous action of both did not provide better protection ${ }^{26}$. Based on the common assumption that IR injury can go through distinct biomolecular pathways, we have proposed to study: (1) the immunochemistry expression of active caspase- 3 and BCL- 2 under the simultaneous action of IPC and PTX, which separately have a favorable effect on intestinal IR damages; and (2) the participation of NOS2 and NOS-3 in the protective effects of IPC and PTX submitted to intestinal IR in rats, with the mRNA expression levels determined from epithelial cells.

\section{Methods}

All animal experiments were carried out in accordance with the recommendations of international legislation on animal protection. The Ethics Committee in Research of the Universidade Federal de São Paulo (UNIFESP) approved the study.

Thirty male Wistar-EPM-1 rats (250$300 \mathrm{~g})$ were purchased from the Center for the Development of Experimental Models for Medicine and Biology (CEDEME, Sao PauloSP, Brazil). The animals were housed under controlled temperature (23]), 50\% air humidity and light conditions with a 12-hour light/ dark cycle. The rats had free access to water and standard pellet chow until 6 hours before the surgical procedures at the Experimental Surgery Department of UNIFESP. The individual cages had dimensions of $40 \mathrm{~cm} \times 30 \mathrm{~cm} \times 25$ $\mathrm{cm}$ and, which were covered with previously autoclaved wood shavings.

All procedures involving anaesthesia and euthanasia were performed according to followed the guidelines of the National Council Animal Experimentation Control (SBCAL/ COBEA - Brazil http://www.cobea.org.br/).

The surgical procedures were performed under intramuscular anesthesia with $80 \mathrm{mg} / \mathrm{kg}$ of ketamine (Dopalen $80 \mathrm{mg} / \mathrm{kg}$, Sespo Indústria Comércio, Paulínea-SP) and 10 $\mathrm{mg} / \mathrm{kg}$ of xylazine (Dopaser $5 \mathrm{mg} / \mathrm{kg}$, Herpate Callieri, Juatuba-MG). The femoral vein was dissected and catheterized for drug infusion of $80 \mathrm{mg} / \mathrm{kg}$ pentoxifylline (Sigma Aldrich, São Paulo-SP) or $0.9 \%$ saline solution in the control group 5 minutes before ischemia. A median abdominal incision was made to identify the superior mesenteric vessels and clamping was done according to the group. The animals were randomly assigned into five groups:

CG - Control group: anesthesia + laparotomy + no clamping of the superior mesenteric artery (timing elapsed 90 minutes).

IR-SS group: saline +30 minutes of ischemia +60 minutes of reperfusion.

IR-PTX group: pentoxifylline + 
30 minutes of ischemia +60 minutes of reperfusion.

IPC-IR-SS group: five minutes of ischemia + five minutes of reperfusion (preconditioning) + saline +30 minutes of ischemia +60 minutes of reperfusion.

IPC-IR-PTX group, five minutes of ischemia + five minutes of reperfusion (preconditioning) + pentoxifylline +30 minutes of ischemia +60 minutes of reperfusion.

At the end of reperfusion, two samples of jejunal segments ( $3 \mathrm{~cm}$ long) were opened longitudinally, gently washed with saline solution, and one of them was wrapped in aluminum foil and frozen in liquid nitrogen. The other one was fixed in $10 \%$ formaldehyde phosphate buffered solution.

\section{Immunohistochemistry (IHC) analysis}

$\mathrm{IHC}$ is a commonly used technique to monitor protein expression and localization in the context of tissue morphology. Tissue samples were fixed in $10 \%$ neutral buffered formaldehyde, embedded in paraffin wax, cut into thin slices $(3 \mu \mathrm{m})$ and mounted onto silanized slides. The sample was deparaffinized and hydrated. Assay procedures were performed according to the manufacturer's instructions. The epitopes were blocked with peroxidase and incubated with a specific first antibody. The primary antibody to verify cell apoptosis was assessed using an anti-active caspase-3 antibody, diluted to $1: 200$ (rabbit heterologous polyclonal antibody ABCAMAB2302, São Paulo-SP). The anti-apoptotic analysis was done using a primary antibody anti-BCL-2, diluted 1:100 (monoclonal mouse antibody, DAKO, M00884, São Paulo-SP). The sections were washed with PBS solution and incubated with biotinylated secondary antibody, avidin-chain enzyme, stained with $\mathrm{DAB}$, and lastly with hematoxylin. The sections were dehydrated conventionally with ethanol, cleared with xylene, and mounted with synthetic resin. A scanner (3D HISTECH ${ }^{\circ}$, Biogen, São Paulo-SP) captured all images of the immunohistochemistry slides. The images were analyzed by specific software Case Viewer (3D HISTECH ${ }^{\circ}$, Biogen, São Paulo-SP; magnification $\times 20, x 40, x 50)$. A perimeter of 1.000 microns was determined on each slide. Within the perimeter, ten villi were randomly selected, and 100 epithelial cells were counted in 10 villi per slice for 5 treatment groups (each group, $\mathrm{N}=6$ ), determining the score of immunohistochemically stained cells 60 villi or 10 villi per slice in 6 groups for each treatment. The data were expressed as mean \pm standard error of the mean $(x \pm$ sem). The difference between the groups was processed by repeated measures analysis of variance and the Bonferroni post hoc comparison test. The results were processed by statistical software Stat Plus the Analyst Soft Inc (version 9.4).

\section{The analysis of mRNA NOS- 2 and NOS-3} expression by $q R T-P C R$

After the storage period, all the tissue samples were prepared for qRT-PCR (LifeTechnologies $^{\mathrm{TM}}$, Grand Island, NY, USA) for NOS-2(iNOS) and NOS-3(eNOS). Initially, for total RNA extraction, the tissue segment was lysed in Trizol (Trizol, Invitrogen ${ }^{\mathrm{TM}}$ ) and homogenized into the extractor (Polytron 3000). Purelink ${ }^{\mathrm{TM}}$ RNA Mini Kit and treatment Pure Link DNAse ${ }^{\mathrm{TM}}$ were used to achieve purification of the total RNA. The samples were subjected to electrophoresis on $1 \%$ agarose gel and reading by Spectrophotometer (NanodroP ${ }^{\circ}$ ) to assess the purity of the samples and their RNA concentrations $(\mu \mathrm{g} / \mathrm{mL})$, respectively. In the reverse transcription of total RNA, for the synthesis of the primary band of complementary DNA or cDNA, SuperScript ${ }^{\mathrm{TM}}$ Vilo $^{\mathrm{TM}}$ Master Mix (Life Technologies ${ }^{\mathrm{TM}}$ - USA) and Reverse Transcriptase were used, and 
the sample was submitted to a GeneAmp 2700 thermocycler (Perkin-Elmer, Inc., Waltham, MA, USA) at $25^{\circ} \mathrm{C}$ temperature for 10 minutes, $42^{\circ} \mathrm{C}$ for 60 minutes and at $85^{\circ} \mathrm{C}$ for 5 minutes. The qRT-PCR reaction occurred from a sample-diluted cDNA and was amplified in $20 \mu \mathrm{L}$ reaction volume distributed also in the wells. The final reaction mixture included the following: primers, 10 pmol; $\mathrm{MgCl}_{2}, 2.5$ $\mathrm{mM}$; Deoxyribonucleosides triphosphate (dNTP) mixture (included in the TaqMan Gene Expression Master Mix kit, Life Technology ${ }^{\mathrm{TM}}$ São Paulo, SP, Brazil), 1 mM; $10 \times$ RNA PCR buffer, 1x; 1x ExTaq ${ }^{\text {TM }}$ DNA polymerase; and 0.5 $\mathrm{U} /$ reaction of AmpliTaq Gold ${ }^{\mathrm{TM}}$ DNA Polymerase (included in the TaqMan Gene Expression Master Mix, Life Technology, São PauloSP). The PCR was performed with an initial denaturation at $95^{\circ} \mathrm{C}$ for 10 minutes, 40 cycles at $95^{\circ} \mathrm{C}$ for 15 seconds and $60^{\circ} \mathrm{C}$ for one minute. Glyceraldehyde 3-phosphate dehydrogenase (GAPDH; TaqMan ${ }^{\text {TM }}$ Endogenous Control, Invitrogen $^{\mathrm{TM}}$ Rn01775763_g1) was used as a "housekeeping gene" (internal control gene) to indicate the amount of cDNA in the sample reference control and the experimental sample in PCR. The target genes were NOS-2 (iNOS) (TaqMan gene expression assay, Invitrogen/ Rn00561646_m1) and NOS-3 (eNOS) (TaqMan gene expression assay, Invitrogen/ Rn02132634_s1). The samples were performed in triplicate. The products were amplified by Step One Plus vs 2.3 software (Applied Biosystems $^{\mathrm{TM}}$ ) to obtain the reading of the amount of amplified cDNA during the reaction. To calculate the mean value of the cycle thresholds $(\mathrm{Ct})$ relative on the reference gene, the value was subtracted from the mean value of the target gene $\mathrm{Ct}$. The $\Delta \Delta \mathrm{Ct}$ calculation was converted into a linear expression from the logarithmic exponential function $(\log 2)^{27}$. Afterward, the results were expressed as the mean, standard error, 95\% confidence interval, and an analysis of variance (ANOVA) and the Bonferroni post hoc test were performed to evaluate significant differences between the means of the experimental groups. The level of rejection of the null hypothesis was set at $5 \%$ ( $p \leq 0.05)$. All calculations were performed using Stat software version $9.4^{\circ}$.

\section{- Results}

Immunohistochemistry analysis with antiactive caspase-3 and anti BCL-2

Analysis of the cell immunoreactivity (Figures $1 \mathrm{~A}$-E) shows the photomicrographs of tissues incubated with activate caspase-3 antibody. The cells of the injured villi were measured, but the phagocytes were not measured. The average of the immunoreactive cells differed between the treatments ( $F=$ $47.2, p<0.05)$. The average scores in the immunoreactive cells in the IR-SS group (64.1 $\pm 3.02 ; 95 \% \mathrm{Cl} 58.1-70.1)$ were significantly higher than those in the CG $(3.37 \pm 0.403 ; 95 \%$ $\mathrm{Cl} 2.56$ - 4.17), IR-PTX $(2.92 \pm 0.488 ; 95 \% \mathrm{Cl}$ 1.94-3.89), IPC-IR-SS (0.72 $\pm 0.128 ; 95 \% \mathrm{Cl} 0.46$ - 0.973), and IPC-IR-PTX $(2.82 \pm 0.373 ; 95 \% \mathrm{Cl}$ 2.07-3.56) groups. There was no significant difference among treatments for the other comparisons (Figure 2). 


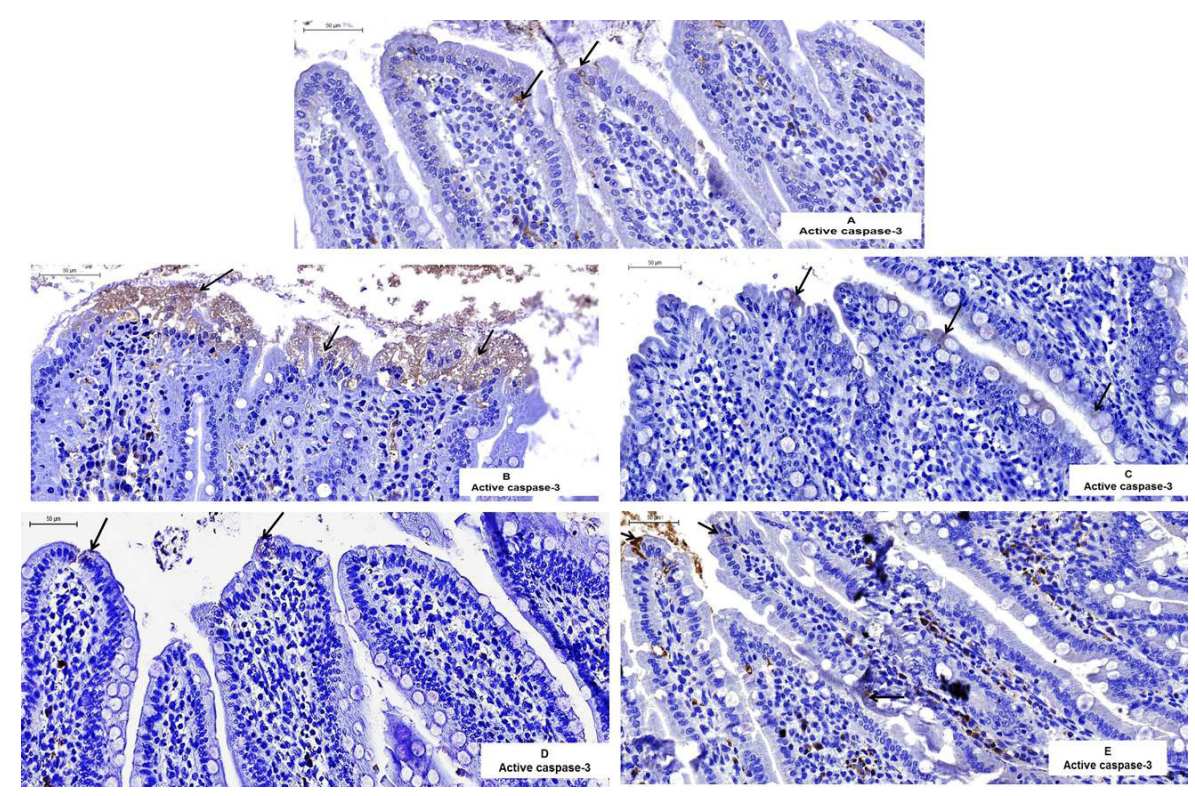

Figure 1 - Photomicrography the histological sections of intestines of rats subjected to active caspase-3 immunostaining and counterstained with hematoxylin. Note the higher immunoreactivity (arrows) in the intestinal villi of the IR-SS group (B). Few active caspase-3-positive cells (arrows) can also be noticed in the CG (A), IR-PTX (C), IPC-IR-SS (D) and IPC-IR-IPTX (E) groups. Scale bare: $50 \mu \mathrm{m}$.

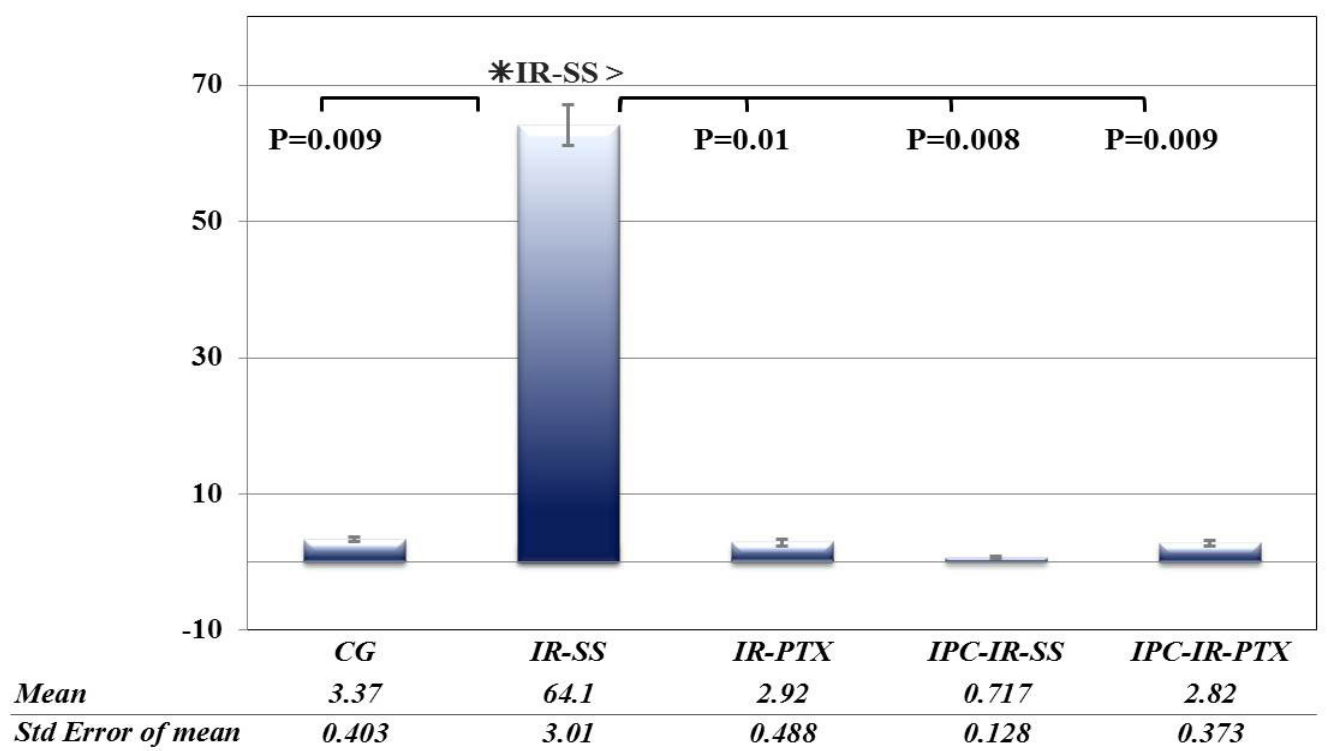

Figure 2 - The expression of active caspase-3 was analyzed, and the difference was determined between groups for the model of repeated measures analysis of variance. $\left({ }^{*}\right)$ The difference was analyzed by pairs; statistically significant values are represented in the chart.

Figures $3 A-E$ show the tissues of rats intestine incubated with BCL-2 antibody. The epithelial stain cells were measured, and the average analysis of the immunoreactive cells differed between the treatments $(F=4.96$; $P=0.0015)$. The immunoreactive cells in the IR-PTX group $(10.5 \pm 0.795 ; 95 \% \mathrm{Cl} 0.832$ 1.1) were significantly higher than those in 
the IR-SS $(5.05 \pm 0.569 ; 95 \% \mathrm{Cl} 1.35-1.61)$, IPC-IR-SS (2.47 $\pm 0.372 ; 95 \% \mathrm{Cl} 0.828-1.04)$, and IPC-IR-PTX ( $2 \pm 0.29 ; 95 \% \mathrm{Cl} 0.736-0.93)$ groups, whereas the averages in the IPC-IR-SS group were significantly lower than those in the CG group (7.95 $\pm 0.78 ; 95 \% \mathrm{Cl} 0.931-1.24)$. There was no significant difference between treatments for the other comparisons (Figure 4).

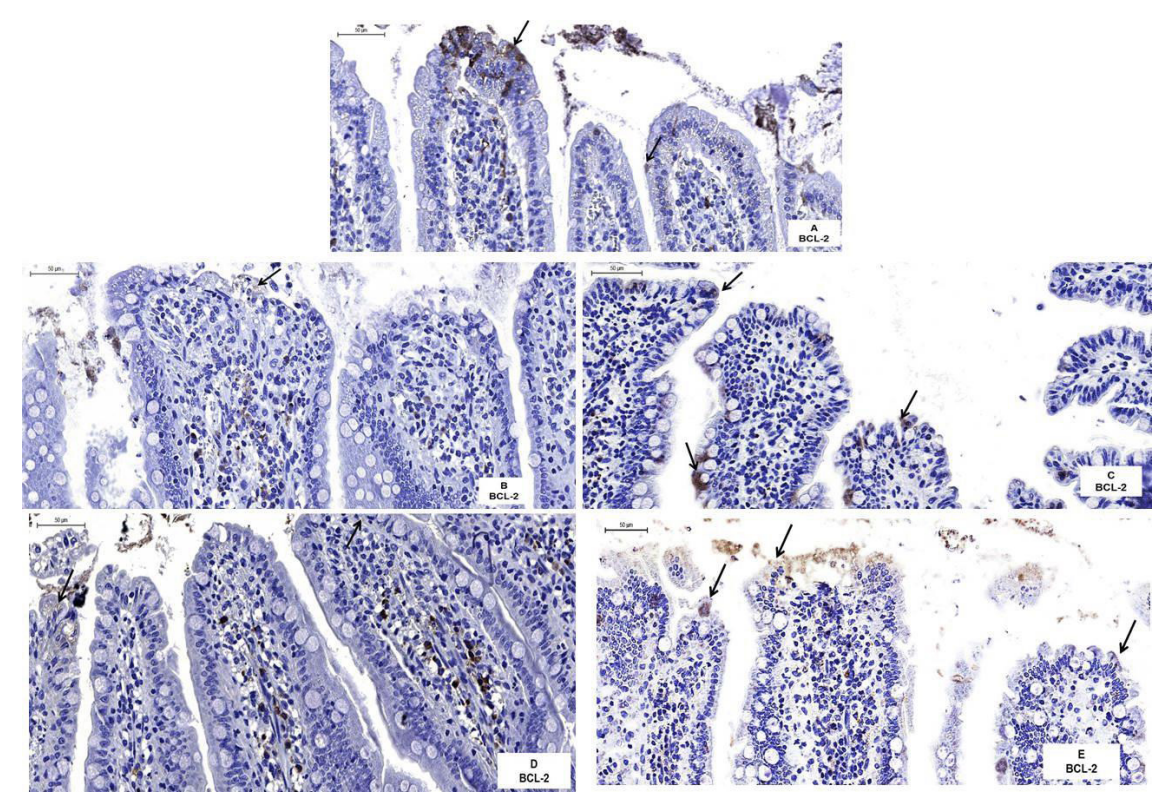

Figure 3 - The photomicrographs of histological sections of rats intestines subjected to BCL-2 immunostaining and counterstained with hematoxylin. Note the higher presence of immunostained epithelial cells (arrows) in the intestinal villi of the CG (A) and IR-PTX (C) groups. Few immunostained epithelial cells (arrows) can also be observed in the IR-SS (B), IPC-IR-SS (D) and IPC-IR-IPTX (E) groups. Scale bare: $50 \mu \mathrm{m}$.

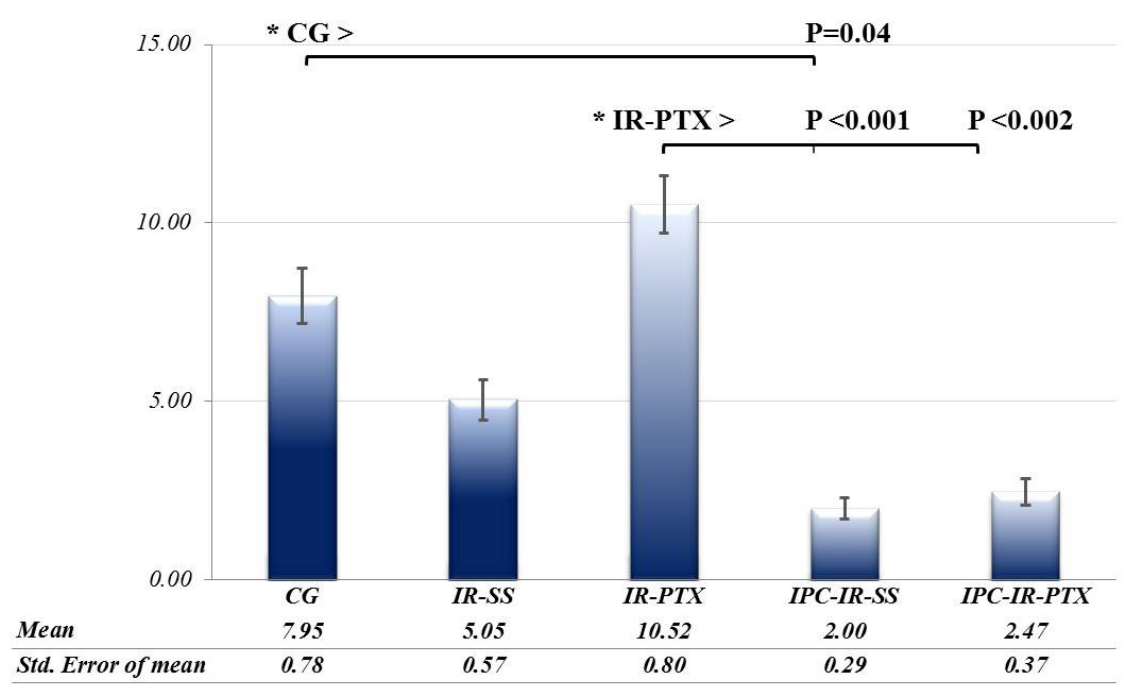

Figure 4 - The expression of BCL-2 was analyzed, and the difference was determined between groups for the model of repeated measures analysis of variance. $\left({ }^{*}\right)$ Differences were analyzed by pairs. Statistically significant values are represented in the graphic. 
Evaluated $m R N A$ expression of NOS-2 and NOS-3 by $q R T-P C R$

The average analysis of expression of NOS-2 differed between the treatments (ANOVA, $\mathrm{F}=8.11, \mathrm{P}=0.0002$ ). The IR-SS group exhibited a significant increase in NOS-2 expression (6.01 \pm 1.34 ; $95 \% \mathrm{Cl}: 2.56$ - 9.47). The NOS-2 level in the control group $(1.38 \pm 0.52$;
$95 \% \mathrm{Cl}: 0.04-2.72)$ was lower than the level in the IR-SS group. The IR-PTX $(2.27 \pm 1.28 ; 95 \% \mathrm{Cl}$ : $-1.02-5.57)$ and IPC-IR-SS $(0.23 \pm 0.10 ; 95 \% \mathrm{Cl}$ : $0.006-0.13$ ) groups significantly reduced NOS2 expression in comparison to the IR-SS group. The combination of procedures in the IPC-IRPTX group $(0.04 \pm 0.01 ; 95 \% \mathrm{Cl}$ : $0.006-0.08)$ drastically reduced NOS-2 expression (Figure 5).

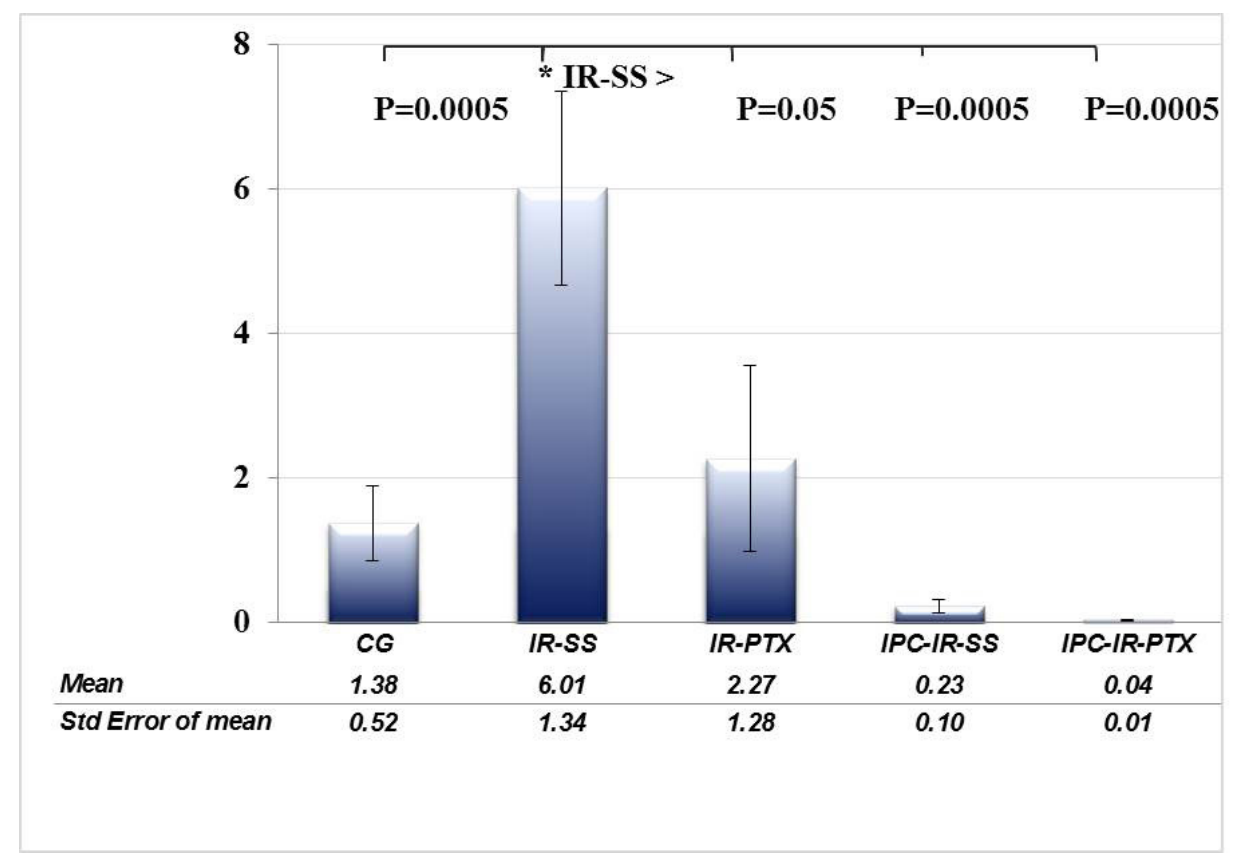

Figure 5 - Data from mRNA expression of NOS-2 by qRT-PCR. Differences among groups were analyzed by ANOVA followed by the Bonferroni test. $\left({ }^{*}\right)$ The differences were analyzed by pairs. Statistically significant values are represented in the graphic.

Our results showed that a significant difference between analysis of average of NOS-3 expressions in the treatments (ANOVA, $\mathrm{F}=5.47, \mathrm{P}=0.0027)$. The NOS-3 expression levels were also increased in the IPC-IR-SS group $(1.61 \pm 0.11 ; 95 \% \mathrm{Cl} 1.32$ - 1.89), IR-SS (1.24 $\pm 0.16 ; 95 \% \mathrm{Cl} 0.83-1.65)$ and the IRPTX group $(1.29 \pm 0.08 ; 95 \% \mathrm{Cl} 1.08$ - 1.49), but these levels were not significantly higher than the levels observed in the control group $(1.10 \pm 0.20$; $95 \% \mathrm{Cl}: 0.58$ - 1.61). However, NOS-3 expression was significantly increased in the IPC-IR-PTX group (1.95 $\pm 0.15 ; 95 \% \mathrm{Cl}: 1.55$ - 2.35) compared with the groups CG, IR-SS, IRPTX (Figure 6). 


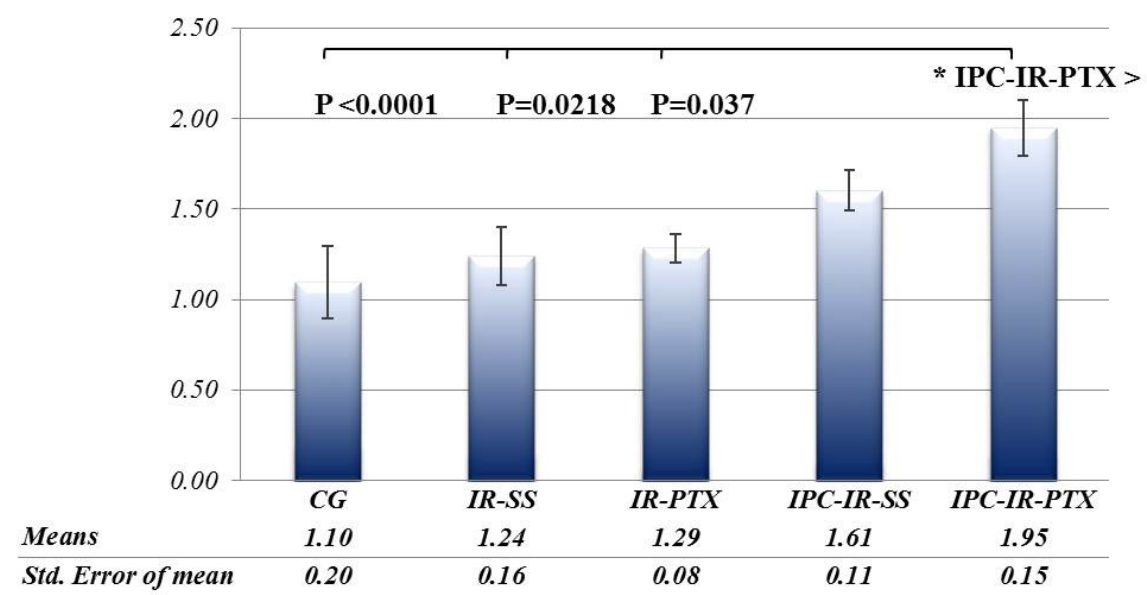

Figure 6 - Data from mRNA expression of NOS-3 by qRT-PCR. Differences among groups were analyzed by ANOVA followed by the Bonferroni test. $\left({ }^{*}\right)$ The differences were analyzed by pairs. Statistically significant values are represented in the graphic.

\section{- Discussion}

With the intent of studying the effects of intestinal ischemia, we have used an animal model in rats that have been subjected to 30 minutes of intestinal ischemia and 60 minutes of reperfusion (IR). We have investigated the effects of pentoxifylline (PTX $-80 \mathrm{mg} / \mathrm{kg}$ ) applied (IV) before the onset of ischemia and/ or ischemic preconditioning (IPC).

This study demonstrates that PTX (80 $\mathrm{mg} / \mathrm{kg}$ ) or IPC can protect against intestinal apoptosis in rats. The immunoreactivity is minimal to active caspase- 3 and present to BCL-2 in the mechanic and pharmacology preconditioning. However, the combination of both did not show an adjuvant effect. In a study of pro-apoptotic genes, the caspase- 3 expression was higher in tissues subjected to $I^{23}$ and lower in tissues subjected to IPC-IR ${ }^{13,15}$. It has been shown that the use of pretreatment with pentoxifylline attuned the expression of active caspase- $3^{23}$ and decreased caspase-3/9 and the c-Jun $\mathrm{N}$-terminal kinase (JNK) signaling pathway ${ }^{8}$.

In intestinal IR, we observed a significant difference in the $\mathrm{BCL}-2$ expression with treatment of IR-PTX compared with the CG, IR and IPC groups. Furthermore, if pentoxifylline could suppress caspase-3/9 and $\mathrm{JNK}^{8}$, this could be interpreted as a significant difference between the anti-apoptotic agents (BCL-2) in this group. In addition, in IPC in the experimental study in the intestine, it could be suggested that the lower stimulation of the apoptotic protein caspase- $3^{6,13}$ would be able maintain the necessary level of BLC-2 $2^{3,4,6,13}$. It provide protections: blocking oxidative damage to cell components and influencing cell membrane transport, altering the distribution of calcium, inhibiting release of cytochrome $\mathrm{C}$ from the mitochondria to the cytoplasm and protecting DNA fragmentation ${ }^{13}$.

Thecelldeath pathways, suchasnecrosis, autophagy, the death receptor apoptotic pathway, post-ischemic inflammation and necroptosis, are operating simultaneously ${ }^{2,5}$. All these pathways could get activated at different times and in certain time gaps. The therapy for prevention of IR, for example, could depend on the mitochondrial apoptotic pathway alone or assessment at a particular time point, or the therapy could release other endogenous substances at the plasma membrane or the 
cytoplasmic pathways, for instance, anion channel, BCL-2 family proteins and JNK, among others molecular pathways $2,5,9,28$.

After IR in the current molecular study, a significant increase in the expression of NOS2 was observed. The expression of nitric oxide synthase (NOS-2 and NOS-3) was evaluated, and the results showed that the mean NOS2 expression in the ischemia and reperfusion group was higher than that for the control group; we also found that the PTX produced a significant reduction of NOS-2. On the other hand, the reduction promoted by IPC, alone or in association with PTX, was intense and almost neutralized the expression of the NOS-2. The NOS-2 enzyme is regulated at the transcriptional level, and its activation is independent of the intracellular calcium concentration ${ }^{2,5,25}$. Experimental research related the antiinflammatory effect and involvement in the control of NOS- $2^{8,9,15}$ and NOS- $3^{28}$ for the action of PTX ${ }^{17-23}$ and IPC 3,4,7,9,11,14-16,25,30. High NO levels associated with inflammatory conditions and activation of NOS-2 have been related to tissue injury ${ }^{3,4,5,9,25}$.

The synthesis of NO by NOS- 2 and NOS3 depends on the induction mechanism, halflife, and the period of stimulation ${ }^{28}$. NO in such conditions may promote tissue damage or beneficial effects ${ }^{2,4,5,9,11,14,15,25,28}$. Analyses of the expression levels of 84 genes encoding proteins that are associated with oxidative stress during 120 minutes of IR in mouse intestine indicated 47 genes, including NOS-2, showed increased expression ${ }^{29}$. Previously, mRNA expression levels of NOS-2 and NOS-3 were evaluated during IR of the superior mesenteric artery (40 minutes of ischemia and 300 minutes of reperfusion) in the rat intestine. The observed effects included increased NO synthesis, decreased mesenteric vascular response, and increased production of the toxic oxidizing species peroxynitrite $(\mathrm{ONOO})^{25}$. In IR, ROS such as $\mathrm{O}_{2-}$ are produced concomitantly with NOS2-derived NO. Both reactive species efficiently react, generating $\mathrm{ONOO}^{-}$, which initiates lipid peroxidation ${ }^{2-6,13,25,29}$. In the other direction, NO binds thiol groups of cysteine of proteins resulting in S- nitrosylation, an important cell signaling pathways ${ }^{9}$.

NO expression is also affected by ischemic preconditioning ${ }^{11}$, by reduction in the generation of pro-inflammatory cytokines and the preservation of blood flow, oxygenation, and mitochondrial function ${ }^{2-5,7,14}$. Furthermore, in the intestine, IPC protection has been associated with inhibition of NOS-2 expression levels $s^{4,14,25,30}$. A study in rat intestines indicated that IPC reduced the expression of leukocyte adhesion proteins, endothelial cell adhesion proteins, and the NF-KB-dependent expression of TNF- $\alpha^{15,16}$. Data from animal model have shown that IPC reduced NO levels in serum and lung after intestinal $I R^{6}$. An investigation on the transcriptional activation of NOS-2 during hepatic hypoxia via intestinal IR found that NFKB activates the transcription of multiple inflammatory genes, including TNF $\alpha$ and IL1 $\beta$. The research suggested that IPC may inhibit NF-kB, p65 activation and decrease liver injury and the infiltration of inflammatory cells ${ }^{13}$. Several studies described the inhibition of NOS-2 expression levels by IPC in heart tissue submitted to IR, and in these conditions, IPC has been associated with cardio protection ${ }^{9}$. These findings, as well as those of the present study, suggest that NOS2 levels decreased in IPC.

NOS-2 mRNA expression levels by the PTX group downregulated TNF-alpha ${ }^{8}$, derived inhibitory effects on NF-kB activity ${ }^{8,18-20,22,23}$. PTX is described to diminished interleukin-1 and interleukin- $6^{17,20,21,23}$, increase intracellular levels of $C A M P$, and exerts its effects through both protein kinase $A$ (PKA) mechanisms ${ }^{17-19}$. Moreover, CAMP and PKA activation have 
been shown to inhibit signaling via the NFk-B pathway and decrease TNF ${ }^{18,19}$ and proinflammatory cytokines ${ }^{21}$.Thus, our hypothesis confirms the beneficial effects listed in these previous findings.

In this study, the NOS-3 expression was significantly higher in the IPC-IR-PTX group than in the IR-PTX, IR-SS and CG groups. In a study of intestines submitted to IR, an increase in $\mathrm{NO}$ and a reduction in intestinal smooth muscle contraction and reactivity to vasoconstrictors was detected ${ }^{25}$. As stated previously, IPC reduced the generation of proinflammatory cytokines, preserving blood flow, oxygenation, and mitochondrial function ${ }^{2-5,7,14}$, and it has been associated with inhibition of NOS-2 expression levels ${ }^{4,14,25,30}$. Preconditioning is triggered by an initial increase in nitric oxide synthesis ${ }^{11}$.

The NOS-3 exerts a function as a vasodilator and an anti-inflammatory mediator $^{28}$. The NOS-3 phosphorylation is regulated up and down and depends on the phosphorylation or dephosphorylation domains at each of the respective sites, the protein-protein interactions, and the bioavailability of cofactors; these can occur simultaneously, with all or combinations of these proceeding to activation. For instance: three domains that contain phosphorylation sites could be inhibiting or stimulating NOS-3; oxygenase, Calmodulin (CaM) and reductase domains. These domains modulate NOS-3 activity and specific stimuli influence multiple pathways such as: AMPK phosphorylates the vasodilator bradykinin and the shear stress, resulting in decreasing NO or increasing NO production, according to the specific activated domain ${ }^{28}$.

Another pathway involving caveolins is associated with the PI3K/Akt signaling pathway ${ }^{28}$. The activity of NOS-3 is regulated by plasma membrane invaginations known as caveolae, which contain $\mathrm{G}$ protein-coupled receptors, heterotrimeric $G$ proteins, tyrosine kinase type receptors, mitogen-activated protein (MAP) kinase pathway proteins, Src tyrosine kinases, and NOS3 ${ }^{9,28}$. The activation of NOS3 also depends on the formation of the $\mathrm{Ca}++$ /calmodulin complex using calcium from the endoplasmic reticulum or the extracellular milieu $^{28}$. Nitric oxide elevation could be from diffusion into the adjacent cell on the smooth muscle of the intestinal vessels and exerting control over the vascular tone ${ }^{25,28}$. NO inhibits the adhesion of platelets and leukocytes to endothelial cells and decreases platelet aggregation; it also participates in the control of the release of a plasminogen-activating factor and its inhibitor, PAI-1, thereby improving hemodynamics ${ }^{17,25}$.

The importance of higherconcentrations of pentoxifylline has been highlighted. The PTX, as a non-selective phosphodiesterase inhibitor, was associated with increases in cAMP levels ${ }^{17-19}$ and an amplified signaling pathway ${ }^{17-19}$. One of the PI3K/Akt signaling pathways that actives the cyclic guanosine monophosphate (cGMP), when in smooth muscle cells constituent of endothelium vessels, raises resistance vessels, which include NOS-3 expression activated via sensitivity to shear stress or other stimuli ${ }^{28}$. The effects that increase vasodilation, as well a consequence of decreases in leukocyte adhesion, increase erythrocyte flexibility ${ }^{17,28}$ and act in the signaling pathways involved in the synthesis of NF-KB expression $8,18-20,22,23$.

The lack of consensus on research protocols related to the best IR duration limits the comparison of our results with those of other studies.

Future studies of IPC and pentoxifylline may focus on other pathways besides the apoptosis inhibition pathways, such as molecular pathways associated with cGMP and CAMP in IR with the ambivalent effect of $\mathrm{NO}$ on these pathways. Besides that NF-KB modulation 
was including the phosphatidylinositol 3 kinase (PI3K/Akt) pathway.

The results of previous studies and this study indicate that pentoxifylline ${ }^{17-23,26}$ and IPC ${ }^{3,4,7,9,11,14-16,25,26,30}$ haverobustprotective effects in the intestine of rats subjected to IR. For this reason, pentoxifylline has anti-inflammatory activity, increases vascular resistance and improves erythrocyte flexibility; this drug can be used in some clinical conditions, including during an acute deficiency in the blood supply and for the attenuation of the adverse effects of organ and tissue transplantation.

The data on the integrity of the rat jejunum mucosa ${ }^{26}$, control the apoptosis and modulation of NOS are relevant to future study of the signaling pathway control of nitric oxide and nitrosylation.

\section{Conclusions}

The IPC and pentoxifylline alone: decrease NOS-2 expression, a compensatory up-regulation of NOS-3 expression and control the apoptosis after intestinal IR injury. When viewed the IPC-PTX and IPC groups, no synergism effects was observed.

\section{References}

1 Grootjans J, Lenaerts K, Buurman WA, Dejong CHC, Derikx JPM. Life and death at the mucosal-luminal interface: new perspectives on human intestinal ischemiareperfusion. World J Gastroenterol. 2016;22(9):2760-70. doi: 10.3748/wjg.v22. i9.2760.

2 Eltzschig HK, Eckle T. Ischemia and reperfusion-from mechanism to translation. Nat Med. 2011;17(11):1391-401. doi: 10.1038/nm.2507.

3 Mallick IH, Yang W, Winslet MC, Seifalian AM. Ischemia-reperfusion injury of the intestine and protective strategies against injury. Dig Dis Sci. 2004;49(9):1359-77. doi: 10.1023/B:DDAS.0000042232.98927.91.

4 Camara-Lemarroy CR. Remote ischemic preconditioning as treatment for nonischemic gastrointestinal disorders: Beyond ischemia-reperfusion injury. World J Gastroenterol. 2014;20:3572-81. doi: 10.3748/wjg.v20.i13.3572.

5 Granger DN, Kvietys PR. Reperfusion injury and reactive oxygen species: the evolution of a concept. Redox Biol. 2015;6:524-51. doi: 10.1016/j.redox.2015.08.020.

6 Wang Z, Ji Y, Wang S, Wang R, Li Z, Kang $A$, Xu H, Shi M, Zhao M. Protective effect of intestinal ischemic preconditioning on ischemia reperfusion-caused lung injury in rats. Inflammation. 2015;38(1):424-32. doi: 10.1007/s10753-014-0047-3.

7 Camprodon RA, Bowles MJ, Pockley AG, de Oca J. Anti-inflammatory effects of ischemic preconditioning on rat small bowel allografts. Transplant Proc. 2014;46(6):2146-9. doi: 10.1016/j.transproceed.2014.06.038.

8 Yang Q, Zheng FP, Zhan YS, Tao J, Tan SW, Liu HL, Wu B. Tumor necrosis factoralpha mediates JNK activation response to intestinal ischemia-reperfusion injury. World J Gastroenterol. 2013;19:4925-34. doi: 10.3748/wjg.v19.i30.4925.

9 Heusch G. Molecular basis of cardioprotection signal transduction in ischemic pre-, post-, and remote conditioning. Circ Res. 2015;116:674-99. doi: 10.1161/ CIRCRESAHA.116.305348.

10 Murry CE, Jennings RB, Reimer KA. Preconditioning with ischemia: a delay of lethal cell injury in ischemic myocardium. Circulation. 1986;74:1124-36. doi: 10.1161/01.CIR.74.5.1124.

11 Hotter G, Closa D, Prados M, FernandezCruz L, Prats N, Gelpi E, Rosello-Catafau J. Intestinal preconditioning is mediated by a transient increase in nitric oxide. Biochem Biophys Res Commun. 1996;222 (1):27-32. doi: 10.1006/bbrc.1996.0692.

12 Taha MO, Miranda-Ferreira R, Chang AC, Rodrigues AM, Fonseca IS, Toral LB, Cardoso MR, Simões MJ, Oliveira-Junior IS, Monteiro HP, Fagundes DJ, Taha NS, Caricati-Neto A. Effect of ischemic preconditioning on injuries caused by ischemia and reperfusion in rat intestine. Transplant Proc. 2012;44:2304-08. doi: 10.1016/j.transproceed.2012.07.056.

13 Xue TM, Tao LD, Zhang J, Xue TM., Tao, LD., Zhang, J, Zhang, PJ, Liu, X., Chen, GF, ZHU, Y.J. Intestinal ischemic preconditioning reduces 
The expression of endothelial and inducible nitric oxide synthase and apoptosis in intestinal ischemia and reperfusion injury under the action of ischemic preconditioning and pentoxifylline Oliveira TRR et al.

liver ischemia reperfusion injury in rats. Mol Med Rep. 2016;13(3):2511-7. doi: 10.3892/ mmr.2016.4817.

14 Sola A, De Oca J, González R, Prats N, Roselló-Catafau J, Gelpí E, Jaurrieta E, Hotter G. Protective effect of ischemic preconditioning on cold preservation and reperfusion injury associated with rat intestinal transplantation. Ann Surg. 2001;234:98-106. doi: 10.1097/00000658200107000-00015.

15 Takeshita M, Tani T, Harada S, Hayashi $H$, Itoh H, Tajima H, Ohnishi I, Takamura H, Fushida S, Kayahara M. Role of transcription factors in small intestinal ischemiareperfusion injury and tolerance induced by ischemic preconditioning. Transplant Proc. 2010;42:3406-13. doi: 10.1016/j. transproceed. 2010.06.038.

$16 \mathrm{Ji} Y Y$, Wang ZD, Wang SF, Wang BT, Yang ZA, Zhou XR, Lei NN, Yue WN. Ischemic preconditioning ameliorates intestinal injury induced by ischemia-reperfusion in rats. World J Gastroenterol. 2015;21:80818. doi: 10.3748/wjg.v21.i26.8081.

17 McCarty MF, O'Keefe JH, DiNicolantonio JJ. Pentoxifylline for vascular health: a brief review of the literature. Open Heart. 2016;3:e000365. doi: 10.1136/ openhrt-2015-000365.

18 Kreth S, Ledderose C, Luchting B, Weis F, Thiel M. Immunomodulatory properties of pentoxifylline are mediated via adenosinedependent pathways. Shock. 2010;34(1):106. doi: 10.1097/SHK.0b013e3181cdc3e2.

19 Costantini TW, Loomis WH, Putnam JG, Kroll L, Eliceiri BP, Baird A, Bansal V, Coimbra R. Pentoxifylline modulates intestinal tight junction signaling after burn injury: Effects on myosin light chain kinase. J Trauma. 2009;66:17-24. doi: 10.1097/ TA.0b013e318191bb1f.

20 Rasslan R, Utiyama EM, Marques GM, Ferreira TC, da Costa VA, de Victo NC, Rasslan S, Montero EF. Inflammatory activity modulation by hypertonic saline and pentoxifylline in a rat model of strangulated closed loop small bowel obstruction. Int J Surg. 2014;12:594-600. doi: 10.1016/j. ijsu.2014.04.007.

21 Carsi JML, Lapena DC, Toledo AH, Fernandez CZ, Pereyra LHT. Pentoxifylline protects the small intestine after severe ischemia and reperfusion. Exp Clin Transplant. 2013;11:250-8. doi: 10.6002/ect.2012.0222. 22 Marques GMN, Rasslan R, Belon AR, Carvalho JG, Felice Neto R, Rasslan S, Utiyama EM, Montero EFS. Pentoxifylline associated to hypertonic saline solution attenuates inflammatory process and apoptosis after intestinal ischemia/reperfusion in rats. Acta Cir Bras. 2014;29:735-41. doi: 10.1590/ S0102-86502014001800007.

$23 \mathrm{Ji} Q$, Zhang $L$, Jia $H, X u$ J. Pentoxifylline inhibits endotoxin-induced NF-kappa B activation and associated production of proinflammatory cytokines. Ann Clin Lab Sci. 2004;34:427-36. PMID: 15648785.

24 Gown AM, Willingham MC. Improved detection of apoptotic cells in archival paraffin sections: immunohistochemistry using antibodies to cleaved caspase 3 . Histochem Cytochem. 2002;50:449-54. doi: 10.1177/00221554020500 0401.

25 Chen CF, Leu FJ, Chen HI, Wang D, Chou SJ. Ischemia/reperfusion-induced low reactivity of the rat superior mesenteric vascular bed is associated with expression of nitric oxide synthases. Transplant Proc. 2006;38:2216-20. doi: 10.1016/j. transproceed.2006.07.022.

26 Oliveira TRR, Oliveira GF, Simões RS, Tikazawa EH, Monteiro HP, Fagundes DJ, Taha $\mathrm{MO}$. The role of ischemic preconditioning and pentoxifylline in intestinal ischemia/ reperfusion injury of rats. Acta Cir Bras. 2017;32(7):559-67. doi: 10.1590/s0102865020170070000007.

27 Livak KJ, Schmittgen TD. Analysis of relative gene expression data using real-time quantitative PCR and the $2-\Delta \Delta$ ct method. Methods. 2001;25(4):402-8. doi: 10.1006/ meth.2001.1262.

28 Shu X, Keller TCt, Begandt D, Butcher JT, Biwer L, Keller AS, Columbus L, Isakson $B E$. Endothelial nitric oxide synthase in the microcirculation. Cell Mol Life Sci. 2015;72:4561-75. doi: 10.1007/s00018015-2021-0.

29 Bertoletto PR, Ikejiri AT, Somaio Neto F, Chaves JC, Teruya R, Bertoletto ER, Taha $\mathrm{MO}$, Fagundes DJ. Oxidative stress gene expression profile in inbred mouse after ischemia/reperfusion small bowel injury. Acta Cir Bras. 2012;27:773-82. doi: 10.1590/ S0102-86502012001100006. 
30 Bjornsson B, Winbladh A, Bojmar L, Sundqvist $T$, Gullstrand $P$, Sandstrom $P$. Conventional, but not remote ischemic preconditioning, reduces iNOS transcription in liver ischemia/reperfusion. World J Gastroenterol. 2014;20:9506-12. doi: 10.3748/wjg.v20.i28.9506.

\section{Acknowledgement}

To Prof. Manuel de Jesus Simões, Chairman of Division of Histology and Structural Biology, Department of Morphology and Genetic, UNIFESP.

\section{Correspondence:}

Profa. Teresinha Regina Ribeiro de Oliveira

Rua João Vicente Ferreira, 1789

79824-030 Dourados - MS Brasil

Tel.: (55 67)3421-7171

gfo1oliveira@gmail.com

Received: July 02, 2017

Review: Sept 05, 2017

Accepted: Oct 08, 2017
Conflict of interest: none

Financial source: Ministério da Educação do Brasil, UFGD

${ }^{1}$ Research performed at Division of Surgical Techniques and Experimental Surgery, Department of Surgery and Therapy Center of Cellular and Molecular (CTCMol), Universidade Federal de São Paulo (UNIFESP), Brazil. 